\title{
Effects of Propofol and Ketamine as Induction Agent to Isoflurane and as Continuous Rate Infusion on Haematobiochemical Parameters in Dogs Premedicated with Glycopyrrolate, Dexmedetomidine and Butorphanol
}

Hitesh Bayan', Kushal Konwar Sarma², Gutti Dhananjaya Rao ${ }^{3}$, Dwijen Kalita ${ }^{2}$, Devojyoti Dutta ${ }^{4}$, Aarabinda Phukan ${ }^{5}$

10.18805/IJAR.B-4535

\begin{abstract}
Background: The study was conducted to evaluate the effect of propofol and ketamine as induction agent to isoflurane and as continuous rate infusion on haematobiochemical parameters in dogs premedicated with glycopyrrolate, dexmedetomidine and butorphenol.

Methods: Twenty four animals were randomly divided into four Groups (A, B, C and D) comprising six animals in each. All the animals were premedicated with glycopyrrolate, dexmedetomidine and Butorphanol. Induction was done with propofol in group A and B and with ketamine in group $C$ and D. Anaesthesia was maintained with isoflurane in group $A$ and $C$ and with propofol and ketamine continuous rate infusion in group $B$ and $D$, respectively. Blood samples were collected at 0 (before premedication), 20, 40 and 60 min (during maintenance) to evaluate different haematobiochmical parameters.

Result: The haemoglobin levels decreased in all the groups but significantly in Group C and Group D. The total erythrocyte count decreased in all the groups with significant change in Group D. The TLC, PCV, platelet, lymphocyte and monocyte count decreased non-significantly. The neutrophil, granulocyte count in all the groups increased non-significantly over the different observation period. The serum glucose and LDH increased significantly in all the groups. The GGT, alkaline phosphatise, BUN and serum creatinine increased non-significantly in all the groups.
\end{abstract}

Key words: Anaesthesia, Continuous rate infusion, Dog, Haematobiochemical, Inhalation.

\section{INTRODUCTION}

General anaesthesia is used in canine patient for various surgical, medical or diagnostic procedures. The general anaesthetic agents used for this purpose should have a wide margin of safety and should not cause any long-term alteration in body homeostasis. Unfortunately, it is well known that almost all anaesthetic agent exhibit evidence of some deleterious effects on the body which may be of short or long duration. Anaesthetic protocols involving single agent have largely been abandoned in favour of protocols that incorporate multiple agents from different classes to achieve sedation, analgesia and muscle relaxation together (Bednarski, 2011). Both inhalation anaesthesia and total intravenous anaesthesia (TIVA) either with repeated bolus injection or continuous infusion are used in small animal surgery depending upon the facilities available and/ or requirement. The choice of anaesthetic agent or its combination and method of administration is mainly targeted to achieve safe and effective anaesthesia without major alteration in body homeostasis. Therefore, the present study was undertaken to evaluate the haematobiochemical effects with isoflurane, propofol and ketamine anaesthesia in glycopyrrolate, dexmedetomidine and butorphanol premedicated dogs.
'Department of Veterinary Surgery and Radiology, College of Veterinary Sciences and Animal Husbandary, Central Agricultural University, Selesih, Aizawl-796 014, Mizoram, India.

2Department of Veterinary Surgery and Radiology, College of Veterinary Science, Assam Agricultural University, Khanapara, Guwahati-781 022, Assam, India.

${ }^{3}$ College of Veterinary Sciences and Animal Husbandry, Central Agricultural University, Jaluki-797 110, Nagaland, India.

${ }^{4}$ Department of Veterinary Physiology, College of Veterinary Science, Khanapara, Guwahati-781 022, Assam, India.

${ }^{5}$ Department of Veterinary Clinical Medicine, Ethics and Jurisprudence, College of Veterinary Science, Khanapara Guwahati-781 022, Assam, India.

Corresponding Author: Hitesh Bayan, Department of Veterinary Surgery and Radiology, College of Veterinary Sciences and Animal Husbandry, Selesih, Aizawl-796 014, Mizoram, India.

Email: hbayan@gmail.com

How to cite this article: Bayan, H., Sarma, K.K., Rao, G.D., Kalita D., Dutta, D. and Phukan, A. (2021). Effects of Propofol and Ketamine as Induction Agent to Isoflurane and as Continuous Rate Infusion on Haematobiochemical Parameters in Dogs Premedicated with Glycopyrrolate, Dexmedetomidine and Butorphanol. Indian Journal of Animal Research. DOI: 10.18805/IJAR.B-4535.

Submitted: 21-05-2021 Accepted: 24-07-2021 Online: 14-08-2021 


\section{MATERIALS AND METHODS}

Twenty four female dogs between 1-6 years of age and irrespective of breeds presented for elective ovariohystere-ctomy were randomly divided into four groups viz. Group $A, B, C$ and $D$ comprising six dogs in each. The animals were fasted for 12 hours and water was withheld for 8 hours prior to the procedure. The surgical site was prepared in all the animals and intravenous PTFE catheter with injection valve and luer lock plug was fixed and slow intravenous fluid (NSS) was administered throughout the procedure.

All the animals were premedicated with glycopyrrolate @ $0.01 \mathrm{mg} / \mathrm{kg} \mathrm{IM}$. Fifteen minutes later, dexmedetomidine $@ 5 \mu \mathrm{g} / \mathrm{kg}$ and Butorphanol @ $0.1 \mathrm{mg} / \mathrm{kg}$ IV were injected to all the animals. Induction of anaesthesia was done with propofol in Group A and B and with ketamine IV till effect in Group C and D. In Group A and C, anaesthesia was maintained with isoflurane using fresh gas flow rate $10 \mathrm{ml} /$ $\mathrm{kg} /$ minute at a higher value of $3-5 \%$ for initial 5 minutes for stabilization and then the vaporizer settings were changed accordingly to maintain surgical plane of anaesthesia. In Group B and D, anaesthesia was maintained with propofol @ 0.2-0.5 mg/kg/min and ketamine @ 0.002-0.02 mg/kg/ min continuous rate of infusion using a syringe infusion pump.

The blood samples $(1 \mathrm{ml})$ were collected in sterile vials containing EDTA for estimation of haematological parameters. The haemoglobin $(\mathrm{g} / \mathrm{d})$ ), packed cell volume (\%), total erythrocyte count (millions/cubic $\mathrm{mm}$ ), total leukocyte count (thousands/cubic $\mathrm{mm}$ ), differential leukocytes count (\%), total granulocyte count (\%) and total platelet count (thousands/cubic $\mathrm{mm}$ ) were estimated at 0 minute (before premedication), 20 minutes, 40 minutes and 60 minutes (during maintenance) using MS4e Automated Haematology Cell Counter.

The blood samples $(2 \mathrm{ml})$ were collected in sterile test tubes and serum was separated for estimation of glucose $(\mathrm{mg} / \mathrm{dl})$, total protein $(\mathrm{g} / \mathrm{dl})$, gamma glutamyl transferase $(\mathrm{U} / \mathrm{L})$, alkaline phosphatase $(\mathrm{U} / \mathrm{L})$, lactate dehydrogenase $(\mathrm{U} / \mathrm{L})$, blood urea nitrogen $(\mathrm{mg} / \mathrm{dll})$ and creatinine $(\mathrm{mg} / \mathrm{dl})$ level at afore mentioned time intervals using DRI-CHEM 4000i automated serum biochemical analyzer.

The data obtained were analyzed using statistical package SPSS version 16. One way ANOVA based on Fisher's Least Significant Difference method was used to determine the significant difference among the different treatments and time intervals. The significant values in the ANOVA were further tested through Duncan multiple range test. Results are presented as mean \pm SE and differences were considered significant when $\mathrm{P}<0.05$.

\section{RESULTS AND DISCUSSION}

The values of haemoglobin did not vary significantly among the groups at $0 \mathrm{~min}$ and at different periods of observation Table 1. The haemoglobin levels recorded at pre induction gradually decreased during the subsequent periods of observation within all the groups. The decrease in haemoglobin values were non-significant in Group $A$ and $B$ whereas in Group $C$ and $D$, the changes were significant $(P<0.05)$. However, all the values were within the physiological limit. Several mechanisms have been suggested for decreased haemoglobin levels during anaesthesia. It might be contributed from vasodilatation at microcirculation level and passage of red blood cells from circulation leading to decreased haemoglobin level in peripheral veins (Naghibi et al., 2002). The splenic pooling of erythrocytes during anaesthesia and shifting of fluids from the extravascular compartment to the intravascular compartment in order to maintain the cardiac output in animals might lead to decrease in haemoglobin level (Skarda and Muir 1996). The decrease in haemoglobin might also be due to haemodilution in response to fluid therapy (Muir et al., 2008).

A gradual non-significant decrease in the total erythrocyte count was observed in Group A, B and C at 20, 40 and 60 min of observation period. In Group D, there was a significant $(P<0.05)$ decrease in total erythrocyte count at $60 \mathrm{~min}$ as compared to the pre-induction values. Similar observations were also recorded by Jadon et al. (2008) with isoflurane, Mazumdar et al. (2015) with dexmedetomidine, Anandmay et al. (2016) with propofol alone and in combination with buprenorphine, Sharma et al. (2014) with ketamine and propofol with isoflurane and Arunkumar et al. (2017) with dexmedetomidine-propofol-isoflurane. The decrease in total erythrocyte count in the present study might be attributed to slpenic pooling of erythrocytes or inter compartmental shifting of fluids in order to maintain the cardiac output (Muir et al., 2008).

Non-significant decreases in TLC from the pre-induction values were recorded in all the groups at the subsequent observation periods. The decreased TLC might be due to the pooling of circulating blood cells in spleen or other reservoirs secondary to decreased sympathetic activity (Wagner et al., 1991) as dexmedetomidine is known to decrease sympathetic activity. It might also be due to enhanced peripheral blood level of adrenaline or noradrenaline which suppresses proliferative response of peripheral blood leucocytes (Felsner et al., 1995).

The PCV showed a non-significant decrease from the pre-induction values within all the groups at different time intervals. The decreased PCV recorded in the present study during the anaesthetic period might be attributed to shifting of fluid from extravascular compartment to intravascular compartment in order to maintain normal cardiac output or slpenic pooling (Muir et al., 2008).

There were no significant changes in the granulocyte count within the groups except a slight rise (non-significant) over the different observation period. The non-significant changes observed in granulocyte count during anaesthesia might be attributable to stress of anaesthesia or surgery. Major surgical procedure together with anaesthetic stress might have caused stress the animal (Sankar et al., 2011) 
Effects of Propofol and Ketamine as Induction Agent to Isoflurane and as Continuous Rate Infusion on Haematobiochemical...

which in turn stimulated the adrenal cortex leading to glucocorticoid production which acted on the circulating granulocyte.

The platelet count decreased non-significantly from the pre-induction values in all the groups. However, the values were within the physiological limit. The non-significant decrease in platelet count observed in the present study might be due to the shifting of fluid from the extravascular compartment to the intravascular compartment (De-Zhang et al., 2012). It might also be due to temporary sequestration of platelets in the liver, spleen and lungs during anaesthesia (Handagama and Feldman 1988).

The neutrophil count did not show any significant changes within the groups except a slight rise as compared to the pre-induction values at subsequent observation period in all the groups and all the values were found to be within the physiological limit. The non-significant changes observed in neutrophil count in the present study might be due to stress of anaesthesia or due to stress of anaesthesia and surgery

Table 1: Effects of anaesthetic treatment on haematological parameters at different observation period in dogs.

\begin{tabular}{|c|c|c|c|c|c|}
\hline Parameters & Group & $0 \mathrm{~min}$ & $20 \mathrm{~min}$ & $40 \min$ & $60 \mathrm{~min}$ \\
\hline \multirow[t]{4}{*}{ Haemoglobin $(g / d l)$} & $A$ & $12.87 \pm 0.38$ & $11.91 \pm 0.47$ & $11.83 \pm 0.24$ & $11.60 \pm 0.51$ \\
\hline & $\mathrm{B}$ & $13.86 \pm 1.09$ & $12.79 \pm 1.02$ & $12.42 \pm 0.82$ & $11.97 \pm 0.89$ \\
\hline & $\mathrm{C}$ & $13.42 \pm 0.36^{b}$ & $12.47 \pm 0.28^{\mathrm{ab}}$ & $12.39 \pm 0.46^{\mathrm{ab}}$ & $12.07 \pm 0.24^{\mathrm{a}}$ \\
\hline & $\mathrm{D}$ & $13.92 \pm 0.51^{\mathrm{b}}$ & $13.12 \pm 0.47^{\mathrm{b}}$ & $12.75 \pm 0.39^{\mathrm{ab}}$ & $11.73 \pm 0.38^{a}$ \\
\hline \multirow[t]{4}{*}{ Total erythrocyte count (millions/cubic mm) } & $A$ & $7.48 \pm 0.34$ & $7.12 \pm 0.30$ & $7.08 \pm 0.28$ & $6.94 \pm 0.19$ \\
\hline & $\mathrm{B}$ & $7.34 \pm 0.22$ & $7.04 \pm 0.37$ & $6.68 \pm 0.28$ & $6.83 \pm 0.24$ \\
\hline & $\mathrm{C}$ & $7.49 \pm 0.28$ & $7.36 \pm 0.14$ & $7.07 \pm 0.20$ & $6.91 \pm 0.18$ \\
\hline & $\mathrm{D}$ & $7.62 \pm 0.33^{b}$ & $7.24 \pm 0.43^{\mathrm{ab}}$ & $6.9 \pm 0.23^{\mathrm{ab}}$ & $6.58 \pm 0.22^{\mathrm{a}}$ \\
\hline \multirow[t]{4}{*}{ Total leukocyte count (thousands/cubic $\mathrm{mm}$ ) } & $A$ & $12.1 \pm 0.67$ & $11.95 \pm 0.50$ & $11.45 \pm 0.39$ & $11.13 \pm 0.33$ \\
\hline & $\mathrm{B}$ & $13.05 \pm 1.12$ & $12.38 \pm 0.63$ & $11.45 \pm 0.68$ & $11.08 \pm 0.42$ \\
\hline & $\mathrm{C}$ & $12.75 \pm 0.76$ & $11.4 \pm 0.53$ & $11.11 \pm 0.41$ & $11.14 \pm 0.38$ \\
\hline & $\mathrm{D}$ & $13.11 \pm 0.78$ & $11.34 \pm 0.49$ & $11.34 \pm 0.53$ & $11.26 \pm 0.76$ \\
\hline \multirow[t]{4}{*}{ Packed cell volume (\%) } & A & $48.58 \pm 2.52$ & $45.5 \pm 2.05$ & $43.45 \pm 1.74$ & $42.94 \pm 1.97$ \\
\hline & $\mathrm{B}$ & $47.81 \pm 2.11$ & $44.77 \pm 2.97$ & $42.61 \pm 1.22$ & $42.41 \pm 2.01$ \\
\hline & $\mathrm{C}$ & $47.61 \pm 2.49$ & $43.8 \pm 1.48$ & $42.77 \pm 1.72$ & $42.51 \pm 2.59$ \\
\hline & $\mathrm{D}$ & $46.93 \pm 2.55$ & $42.6 \pm 2.57$ & $42.14 \pm 2.19$ & $41.36 \pm 1.45$ \\
\hline \multirow[t]{4}{*}{ Granulocyte count (\%) } & A & $71.23 \pm 1.79$ & $71.71 \pm 1.50$ & $72.27 \pm 2.85$ & $73.40 \pm 2.54$ \\
\hline & $\mathrm{B}$ & $69.90 \pm 1.76$ & $70.66 \pm 2.29$ & $71.81 \pm 2.68$ & $72.43 \pm 3.34$ \\
\hline & C & $71.90 \pm 1.81$ & $72.97 \pm 1.77$ & $73.79 \pm 2.18$ & $74.08 \pm 2.80$ \\
\hline & $\mathrm{D}$ & $70.87 \pm 2.17$ & $71.59 \pm 1.45$ & $72.85 \pm 2.46$ & $73.37 \pm 3.23$ \\
\hline \multirow[t]{4}{*}{ Platelet count (thousands/cubic mm) } & $A$ & $248.83 \pm 18.65$ & $240.83 \pm 16.23$ & $237.83 \pm 18.67$ & $236.17 \pm 16.90$ \\
\hline & $\mathrm{B}$ & $254.83 \pm 12.68$ & $250.17 \pm 11.52$ & $242.17 \pm 22.31$ & $238.5 \pm 14.36$ \\
\hline & $\mathrm{C}$ & $251.67 \pm 22.44$ & $243.83 \pm 23.45$ & $236.17 \pm 14.79$ & $235.33 \pm 14.58$ \\
\hline & $\mathrm{D}$ & $245.83 \pm 21.04$ & $236.5 \pm 19.53$ & $230.83 \pm 14.63$ & $229.83 \pm 15.58$ \\
\hline \multirow[t]{4}{*}{ Neutrophil count (\%) } & $A$ & $67.04 \pm 3.54$ & $67.86 \pm 1.68$ & $68.57 .66 \pm 1.42$ & $69.41 \pm 1.98$ \\
\hline & $\mathrm{B}$ & $66.24 \pm 3.06$ & $67.12 \pm 3.47$ & $68.45 \pm 2.14$ & $68.96 \pm 2.59$ \\
\hline & $\mathrm{C}$ & $65.37 \pm 2.48$ & $67.24 \pm 1.89$ & $67.87 \pm 1.39$ & $69.21 \pm 1.69$ \\
\hline & $\mathrm{D}$ & $66.54 \pm 1.98$ & $67.12 \pm 2.31$ & $68.25 \pm 1.43$ & $70.09 \pm 2.00$ \\
\hline \multirow[t]{4}{*}{ Lymphocyte count (\%) } & $A$ & $22.23 \pm 2.09$ & $20.62 \pm 1.37$ & $19.72 \pm 1.93$ & $19.11 \pm 1.61$ \\
\hline & $\mathrm{B}$ & $23.89 \pm 1.95$ & $22.218 \pm 1.49$ & $21.21 \pm 1.31$ & $21.12 \pm 1.87$ \\
\hline & $\mathrm{C}$ & $24.69 \pm 3.15$ & $23.43 \pm 2.31$ & $22.74 \pm 2.06$ & $21.89 \pm 3.31$ \\
\hline & $\mathrm{D}$ & $24.76 \pm 2.92$ & $23.15 \pm 2.12$ & $22.32 \pm 2.59$ & $21.78 \pm 1.84$ \\
\hline \multirow[t]{4}{*}{ Monocyte count (\%) } & $A$ & $3.14 \pm 0.41$ & $3.04 \pm 0.37$ & $3.01 \pm 0.35$ & $2.92 \pm 0.41$ \\
\hline & $\mathrm{B}$ & $3.22 \pm 0.24$ & $3.20 \pm 0.22$ & $3.19 \pm 0.20$ & $3.13 \pm 0.14$ \\
\hline & $\mathrm{C}$ & $3.21 \pm 0.28$ & $3.12 \pm 0.18$ & $3.11 \pm 0.21$ & $3.02 \pm 0.15$ \\
\hline & $\mathrm{D}$ & $3.13 \pm 0.28$ & $3.05 \pm 0.25$ & $3.02 \pm 0.24$ & $2.96 \pm 0.24$ \\
\hline \multirow[t]{4}{*}{ Eosinophil count (\%) } & $A$ & $2.26 \pm 0.13$ & $2.12 \pm 0.12$ & $2.04 \pm 0.18$ & $2.05 \pm 0.10$ \\
\hline & B & $2.16 \pm 0.13$ & $2.05 \pm 0.12$ & $2.07 \pm 0.10$ & $1.98 \pm 0.19$ \\
\hline & $\mathrm{C}$ & $2.23 \pm 0.12$ & $2.14 \pm 0.16$ & $2.19 \pm 0.13$ & $2.11 \pm 0.17$ \\
\hline & $\mathrm{D}$ & $2.17 \pm 0.12$ & $2.12 \pm 0.08$ & $1.96 \pm 0.08$ & $2.06 \pm 0.08$ \\
\hline
\end{tabular}

Values in the same row with different superscripts differ significantly $(P<0.05)$. 
both. Major surgical procedure together with anaesthetic treatments might have caused stress in the animals (Sankar et al., 2011). The stress might have stimulated the adrenal cortex leading to glucocorticoid production which acted on the circulating neutrophil.

The lymphocyte count decreased non-significantly within a group from the pre-induction values at subsequent time intervals and the values were within the physiological limit. The non-significant changes observed in the present study might be due to shifting of fluid from the extravascular compartment to the intravascular compartment (De-Zhang et al., 2012), intravenous fluid administration throughout the anaesthetic period and also might be due to splenic vasodilatation. Molinan (2006) opined that opioids may suppress lymphocyte via an indirect mechanism operating through central nervous system. Lymphoid organs contain rich supply of sympathetic nerve fibres permitting norepinephrine to influence lymphocyte activity (Straub et al., 1989).

The monocyte count did not vary significantly among the groups. There was a non-significant decrease in monocyte count towards the end of the observation period within groups. The non-significant changes observed in the present study might be due to intravenous fluid administration throughout the anaesthetic period and also might be due to splenic vasodilatation.

The eosinophil count did not vary significantly at $0 \mathrm{~min}$ and subsequent observation periods among the groups as well as within the groups. Similar observation were also recorded by Sharma et al. (2014) with xylazine-propofol, Jena et al. (2014) with dexmedetomidine-propofol and xylazine-propofol, Suresha et al. (2012) with diazepampropofol in dogs.

The serum glucose level showed a significant $(P<0.05)$ increase from pre-induction values in all the groups at the subsequent observation period Table 2 . The increase in serum glucose level might be due to alpha-2 adrenergic inhibition. The alpha-2 adrenergic agonist have been reported to induce an increase in serum glucose by suppressing insulin release, stimulating glucagon release or both in alpha and beta cells of pancreas (Angel and Lager 1988). It might also be due to decreased membrane transport of glucose and activation of the sympathoadrenal system releasing adrenaline which in turn mobilized glycogen from liver during anaesthesia (Kumar et al., 2016).

The values of serum total protein level did not vary significantly among the groups but showed a non-significant decrease within the groups over time. Similar findings were

Table 2: Effects of anaesthetic treatment on biochemical parameters at different observation period in dogs.

\begin{tabular}{|c|c|c|c|c|c|}
\hline Parameters & Group & $0 \mathrm{~min}$ & $20 \mathrm{~min}$ & $40 \mathrm{~min}$ & $60 \mathrm{~min}$ \\
\hline \multirow[t]{4}{*}{ Glucose (mg/dl) } & A & $81.17 \pm 4.37^{a}$ & $87.67 \pm 4.63^{\mathrm{ab}}$ & $95.83 \pm 4.55^{\mathrm{b}}$ & $101.17 \pm 4.32^{b}$ \\
\hline & $\mathrm{B}$ & $89.67 \pm 5.12^{\mathrm{a}}$ & $98.17 \pm 3.84^{\mathrm{ab}}$ & $103.5 \pm 4.08^{b}$ & $107.17 \pm 4.11^{b}$ \\
\hline & $\mathrm{C}$ & $77.33 \pm 4.70^{\mathrm{a}}$ & $89.83 \pm 6.89^{a b}$ & $94.83 \pm 6.77^{b}$ & $99.67 \pm 2.73^{b}$ \\
\hline & $\mathrm{D}$ & $85.83 \pm 6.39^{a}$ & $94.83 \pm 7.04^{\mathrm{ab}}$ & $99.33 \pm 6.12^{\mathrm{ab}}$ & $108.33 \pm 5.14^{b}$ \\
\hline \multirow[t]{4}{*}{ Total protein $(\mathrm{g} / \mathrm{dl})$} & A & $6.42 \pm 0.30$ & $6.18 \pm 0.25$ & $6.08 \pm 0.28$ & $6.02 \pm 0.30$ \\
\hline & B & $6.78 \pm 0.29$ & $6.44 \pm 0.34$ & $6.39 \pm 0.18$ & $6.35 \pm 0.17$ \\
\hline & C & $6.37 \pm 0.25$ & $6.12 \pm 0.19$ & $6.12 \pm 0.24$ & $6.02 \pm 0.21$ \\
\hline & $\mathrm{D}$ & $6.5 \pm 0.25$ & $6.3 \pm 0.22$ & $6.12 \pm 0.24$ & $6.12 \pm 0.17$ \\
\hline \multirow[t]{4}{*}{ Gamma glutamyl transferase (U/L) } & A & $2.67 \pm 0.26$ & $2.74 \pm 0.22$ & $2.78 \pm 0.23$ & $2.86 \pm 0.20$ \\
\hline & $\mathrm{B}$ & $2.77 \pm 0.29$ & $2.89 \pm 0.29$ & $2.97 \pm 0.35$ & $3.04 \pm 0.33$ \\
\hline & $\mathrm{C}$ & $2.53 \pm 0.22$ & $2.64 \pm 0.23$ & $2.78 \pm 0.22$ & $2.92 \pm 0.11$ \\
\hline & $\mathrm{D}$ & $2.78 \pm 0.42$ & $2.84 \pm 0.28$ & $2.98 \pm 0.20$ & $3.05 \pm 0.16$ \\
\hline \multirow[t]{4}{*}{ Alkaline phosphatase (U/L) } & A & $76.50 \pm 3.95$ & $78.33 \pm 3.55$ & $79.5 \pm 3.42$ & $78.67 \pm 3.26$ \\
\hline & B & $71.33 \pm 5.25$ & $74.33 \pm 5.24$ & $75.17 \pm 5.58$ & $77.87 \pm 4.91$ \\
\hline & $\mathrm{C}$ & $75.33 \pm 4.92$ & $77.23 \pm 4.76$ & $77.67 \pm 4.22$ & $79.57 \pm 4.54$ \\
\hline & $\mathrm{D}$ & $74.5 \pm 5.71$ & $76.83 \pm 5.22$ & $78.33 \pm 4.51$ & $81.33 \pm 4.75$ \\
\hline \multirow[t]{4}{*}{ Blood urea nitrogen $(\mathrm{mg} / \mathrm{dl})$} & $A$ & $7.23 \pm 0.81$ & $7.42 \pm 0.90$ & $7.48 \pm 0.86$ & $7.57 \pm 0.89$ \\
\hline & $\mathrm{B}$ & $8.13 \pm 0.58$ & $8.47 \pm 0.72$ & $8.62 \pm 0.66$ & $9.15 \pm 0.72$ \\
\hline & C & $6.77 \pm 0.37$ & $7.15 \pm 0.37$ & $7.53 \pm 0.31$ & $7.68 \pm 0.40$ \\
\hline & $\mathrm{D}$ & $7.45 \pm 0.33$ & $7.85 \pm 0.39$ & $7.88 \pm 0.49$ & $8.03 \pm 0.54$ \\
\hline \multirow[t]{4}{*}{ Creatinine $(\mathrm{mg} / \mathrm{dl})$} & $A$ & $1.26 \pm 0.16$ & $1.42 \pm 0.15$ & $1.58 \pm 0.14$ & $1.57 \pm 0.11$ \\
\hline & $\mathrm{B}$ & $1.38 \pm 0.19$ & $1.57 \pm 0.17$ & $1.63 \pm 0.18$ & $1.72 \pm 0.08$ \\
\hline & C & $1.29 \pm 0.17$ & $1.43 \pm 0.16$ & $1.57 \pm 0.15$ & $1.68 \pm 0.14$ \\
\hline & $\mathrm{D}$ & $1.37 \pm 0.15$ & $1.62 \pm 0.14$ & $1.68 \pm 0.17$ & $1.73 \pm 0.21$ \\
\hline \multirow[t]{4}{*}{ Lactate dehydrogenase (U/L) } & A & $128.33 \pm 7.41^{\mathrm{a}}$ & $138.17 \pm 8.00^{\mathrm{ab}}$ & $149.17 \pm 5.86^{\mathrm{bc}}$ & $160.83 \pm 4.50^{\circ}$ \\
\hline & $\mathrm{B}$ & $124.5 \pm 6.61^{\mathrm{a}}$ & $136.67 \pm 6.76^{\mathrm{ab}}$ & $147.17 \pm 5.71^{\mathrm{bc}}$ & $157.67 \pm 4.19$ \\
\hline & $\mathrm{C}$ & $133.83 \pm 3.48^{a}$ & $145.5 \pm 5.56^{\mathrm{ab}}$ & $149.5 \pm 6.02^{\mathrm{ab}}$ & $159.17 \pm 5.78^{b}$ \\
\hline & $\mathrm{D}$ & $130.17 \pm 8.98^{a}$ & $144.67 \pm 6.92^{\mathrm{ab}}$ & $153.17 \pm 6.92^{b}$ & $159.83 \pm 5.36^{b}$ \\
\hline
\end{tabular}

Values in the same row with different superscripts differ significantly $(P<0.05)$. 
also reported by Jena et al. (2014) with propofol, Kumar et al. (2016) with dexmedetomidine-ketamine, Sharma et al. (2014) with ketamine and halothane along with butorphanol, dexmedetomidine and xylazine in dogs. The non-significant reduction of serum total protein within the normal range during anaesthesia indicated minimal effect of the anaesthetics on the liver. The reduction might be attributable to the inter-compartmental fluid shift (Wagner and Hitchcliff 1991).

The values of gamma glutamyl transferase level did not vary significantly at $0 \mathrm{~min}$ and at different observation period among the groups. The gamma glutamyl transferase values were slightly higher than the pre-induction values at the end of the observation period in all the groups. All the changes recorded were within the physiological limit. The GGT is more sensitive than ALT and AST in detecting liver and gall bladder affections and changes in GGT occur faster than other liver enzymes and persists longer (Guzel et al., 2006). These findings were suggestive of minimum effects on the liver and gall bladder during the anaesthetic period as Braun et al. (1983) opined that increase in serum GGT level caused by transient cholestasis.

The alkaline phosphatase values did not vary significantly as compared to the pre-induction values at the subsequent observation periods in all the groups and the values were within the physiological limit. Non-significant changes in the alkaline phosphates level in the present study indicated minimal affects of the anaesthetic agents on liver, intestine, kidney and bone (Komnenou et al., 2005)

The values of blood urea nitrogen level did not vary significantly at $0 \mathrm{~min}$ and at different observation periods among the groups. The blood urea nitrogen values were slightly higher than the pre-induction values at different observation periods in all the groups; however, the changes were within the physiological limit. The slight non-significant increase in urea nitrogen might be due to the effect of different anaesthetic drugs on renal blood flow and consequent decrease in glomerular filtration rate, changes in the cardiovascular and neuroendocrine activity that affects transiently on renal functions (Surbhi et al., 2010). The increased hepatic urea production from amino acid degradation could also account for the increased urea nitrogen values during maximum depth of anaesthesia.

The serum creatinine values increased non-significantly in all the groups as compared to the pre-induction values. In Group B and D, the values were slightly higher than in Group A and C. However, the changes were within the physiological limit. The non significant increase in serum creatinine might be due to the effect of different anaesthetic drugs on renal blood flow, changes in the cardiovascular and neuroendocrine activity that affects transiently on renal functions (Surbhi et al., 2010). The non-significant changes in creatinine indicated that the anaesthetic combinations in the present study had a minimal affect on the renal system. Dexmedetomidine preserves blood supply to vital organs like brain, heart, liver and kidney at the expense of organs like skin and pancreas (Lawrence et al., 1996). This might be responsible for maintaining creatinine values within normal limit in all the groups.

The $L D H$ values increased significantly $(P<0.05)$ in Group $A, C$ and $D$ within the group till the end of the observation periods. The rises in LDH were highly significant $(P<0.01)$ in group $B$. However, the LDH values in all the groups were in the normal limit. Similar observations were also reported by Yuan et al. (2012) with isoflurane and savoflurane, Khandekar et al. (2015) with both propofol and halothane, Dinesh et al. (2018) with propofol-isoflurane and ketamine-isoflurane in dogs. The LDH values in all the groups which remained within the physiological limit indicated minimum tissue or cellular damage (Camkerten, 2013) reflecting the safety of the anaesthetic agents.

\section{CONCLUSION}

The changes in haematobiochemical parameters were within the physiological limit and all the anaesthetic combinations were found to be safe in dog in terms of haematobiochemical parameters.

\section{REFERENECES}

Anandmay, A.K., Dass, L.L., Sharma, A.K. and Gupta, M.K. (2016). Haemato-biochemical changes following administration of propofol in combination with buprenorphine in atropinized dogs. Journal of Animal Research. 6(3): 531-536.

Angel, I. and Langer, S.Z. (1988). Adrenaergic induced hyperglycaemia in anaesthetized rats: Involvement of peripheral alpha-2 adrenoceptors. Eur. J. Pharmacol. 154(2): 191-196.

Arunkumar, S., Dilipkumar, D. and Shivaprakash, B.V. (2017). Haemodynamic and haematobiochemical evaluation of dexmedetomidine, xylazine and triflupromazine as preanaesthetics with propofol-isoflurane anaesthesia for various surgeries in dogs. Indian J. Vet. Surg. 38(1): 47-48.

Bednarski (2011). Anaesthesia management of dogs and cats. In: Essentials of Small Animal Anaesthesia and Analgesia. [Grimm, K.A., Tranquilli, W.J. and Lamont, L.A. (Eds.)], Wiley-Blackwell. Pp. 279.

Braun, J.P., Benard, P., Burgat, V. and Rico, A.G. (1983). Gamma glutamyl transferase in domestic animals. Vet. Res. Commun. 6: 77-90.

Camkerten, I., Sindak, N., Ozkkurt, N., Ipek, H., Biricik, S and Sahin, T. (2013). Effect of ketamine-xylazine anesthesia on some hematological and serum biochemical values of bozova greyhounds. Harran. Univ. Vet. Fak. Derg. 2(1): 27-31.

De-Zhang, L.U., Hong-gang, F., Sheng, J., Luan-song, Z., Kun, M., Shi-ming, Y.U., Li-juan, T. and Hong-bin, W. (2012). Cardiopulmonary, biochemical and hematological effects of the tiletamine/ zolazepam-xylazine tramadol combination to provide anesthesia in miniature pigs. J. Integrative Agricult. 11(8): 1340-1346.

Dinesh, Bisla, R.S., Tayal, R., Chaudhary, R.N. and Kumar, A. (2018). Comparative evaluation of efficacy and safety of two balanced anaesthetic protocols in female dogs undergoing mammary tumor resection. The Pharma Innovation Journal. 7(8): 286-292. 
Felsner, P., Hofer, D., Rinner, I., Porta, S., Korsatko, W. and Schauenstein, K. (1995). Adrenergic suppression of peripheral blood $\mathrm{T}$ cell reactivity in the rat due to activation of $\alpha$-2-receptors. J. Neuroimmunol. 57: 27-34.

Guzel, O., Inal, G., Cirakli, Z.T., Eraslan, E. and Aktas, M. (2006). Effect of propofol and sevoflurane anesthesia on some physiological and biochemical parameters in rabbits. Medycyna Wet. 62(12): 1383.

Handagama, P. and Feldman, F. (1988). Thrombocytopenia and drugs. The Veterinary Clinics of North America: Small Animal Practice. 18(1): 15-65.

Jadon, N.S., Kumar, S., Kandpal, M., Sharma, V.K. and Thathoo, A.K. (2008). Comparative evaluation of isoflurane and sevoflurane anaesthesia in puppies. Ind. J. Vet. Surg. 29(2): 110-111.

Jena, B., Das, J., Nath, I., Sardar, K.K., Sahoo, A., Beura, S.S. and Painuli, A. (2014). Clinical evaluation of TIVA using xylazine or dexmedetomidine with propofol in surgical management of canine patients. Veterinary World. 7(9): 671-680.

Khandekar, G.S., Sarkate, S.B. and Bendale, K.R. (2015). Physiological and haematobiochemical changes under propofol and halothane anaesthesia in dogs undergoing laparoscopic procedures. Indian J. Vet. Surg. 36(1):17-20.

Komnenou, A., Karayannopoulou, M., Polizopoulou, Z.S., Constantinidis, T.C. and Dessiris, A. (2005). Correlation of serum alkaline phosphatase activity with the healing process of long bone fracture in dogs. Veterinary Clinical Pathology. 34(1): 35-38.

Kumar, S.S. H, Dass, L.L., Sharma, A.K., Gupta, M.K. and Roy, B.K. (2016). Clinical, anaesthetic and biochemical effects of Lycopersicum esculentum (Tomato) as prenaesthetic to propofol anaesthesia in dogs. Indain J. Vet. Surg. 37(1): 36-39.

Lawrence, C.J., Prinzen, F.W. and Lange, S. (1996). The effect dexmedetomidine on nutrient organ flow. Anesth. Analg. 83(6): 1160-1165.

Mazumdar, H., Sarma, B., Sarma, K.K. and Mazumdar, A. (2015). Haemato-biochemical effects of dexmedetomidine in dog. International Journal of Recent Scientific Research. 6(7): 5301-5303.

Molinan, P.E. (2006). Opioids and opiates. Analagesia with cardiovascular haemodynamics and immune implications and critical illness. J. Intern. Med. 259: 138-154.
Muir, W., Lerche, P., Wiese, A., Nelson, L., Pasloske, K., Whittem, T. (2008). Cardiorespiratory and anaesthetic effects of clinical and supra clinical doses of alfaxalone in dogs. Veterinary Anaesthesia and Analgesia. 35: 451-462.

Naghibi, K.H., Yaraghi, A. and Adibi, P. (2002). Haemoglobin and haematocrit changes during uncomplicated anesthesia: General anaesthesia and local anaesthesia. J. Res. Med. Sci. 7(4): 9.

Sankar, P., Jastin William, B., Rao, G.D., Prathaban, S., Suresh Kumar, R. and Leela, V. (2011). Cardiopulmonary and haematobiochemical alterations during ketamine or propofol anaesthesia in acepromazine-xylazine premedicated horses. Indian J. Vet. Surg. 32(1): 23-26.

Sharma, R., Kumar, A., Kumar, A., Sharma, S.K., Sharma, A. and Tewari, N. (2014). Comparison of xylazine and dexmedetomidine as a premedicant for general anaesthesia in dogs. Indian J. Anim. Sc. 84(1): 8-12.

Skarda, R.T. and Muir, W.W. (1996). Comparison of antinociceptive, cardiovascular and respiratory effects, head ptosis and position of pelvic limbs in mares after caudal epidural administration of xylazine and detomidine hydrochloride solution. Am. J. Vet. Res. 51: 556-560.

Straub, R.H. and Scholmerich, J. (1989). Dialogue between the CNS and the immune system in lymphoid organs. Immunology Today. 19: 409-413.

Surbhi, Kinjavdekar, P., Amarpal, Aithal, H.P., Pawde, A.M., Pathak, M. and Borena, B.M. (2010). Physiological and biochemical effects of medetomidine-butorphanol-propofol anaesthesia in dogs undergoing orthopaedic surgery. Ind. J. Vet. Surg. 31(2): 101-104.

Suresha, L., Ranganath, B.N., Vasanth, M.S. and Ranganath, L. (2012). Haemato-biochemical studies on triflupromazine $\mathrm{HCL}$ and diazepam premedication for propofol anaesthesia in dogs. Vet. World. 5(11): 672-675.

Wagner, A.E. and Hitchcliff, K.W. (1991). Cardiovascular effects of xylazine and detomidine in horses. Am. J. Vet. Res. 52(5): 651-657.

Yuan, Z., Liu, J., Liang, X. and Lin, D. (2012). Serum biochemical indicators of hepatobiliary function in dogs following prolonged anaesthesia with sevoflurane or isoflurane. Vet. Anaesth. Analg. 39(3): 296-300. 